\title{
Pilot Study of Probe-based Confocal Laser Endomicroscopy with Fluorescein-dripping Method for Liver Tumors
}

\author{
MASAKI MORIMOTO, SOICHIRO HONJO, TERUHISA SAKAMOTO, EI UCHINAKA, \\ MASATAKA AMISAKI, YOSUKE ARAI, MANABU YAMAMOTO, YOJI FUKUMOTO, TOMOHIRO OSAKI, \\ NARUO TOKUYASU, KEIGO ASHIDA, HIROAKI SAITO and YOSHIYUKI FUJIWARA
}

Department of Surgery, Division of Surgical Oncology, Faculty of Medicine, Tottori University, Yonago, Japan

\begin{abstract}
Background/Aim: Probe-based confocal laser endomicroscopy ( $p C L E)$ is a novel diagnostic technique that can provide real-time imaging of tissues at the cellular level. In this study, pCLE was applied to liver tumors and its diagnostic value was evaluated. Patients and Methods: Ten patients who underwent hepatectomy for liver tumors were evaluated with pCLE from February to May 2017. Immediately after liver resection, $p C L E$ evaluation was performed in the operating room as an ex vivo study. The newly-adopted "fluorescein-dripping method" was used for the observation. Results: First, the optimal fluorescein exposure time for the surface of liver was defined in the fluorescein-dripping method. Next, the distinctive findings in the cancerous region were investigated. The characteristic appearance of irregular arrangements of concentrated cells under fluorescein dripping was observed in six of seven hepatocellular carcinoma (HCC) tumors. Conclusion: In all $H C C$ specimens, discrimination of the cancerous region from normal liver was possible with $p C L E$.
\end{abstract}

Liver cancer, such as hepatocellular carcinoma (HCC) or intrahepatic cholangiocarcinoma, is the second leading cause of cancer-related death worldwide (1). Metastatic liver tumors originating from various organs are also commonly observed. Liver resection should be selected in some patients because of its high likelihood of cure. In patients with liver cancer or metastatic liver tumors (especially from colorectal cancer), the surgical margin is an important prognostic factor for curative resection $(2,3)$. However, no reliable

Coorespondence to: Dr. Soichiro Honjo, Department of Surgery, Division of Surgical Oncology Faculty of Medicine, Tottori University, 36-1 Nishi-cho, Yonago 683-8504, Japan. Tel: +81 859386567, Fax: +81 859386569, e-mail: honjo@tottori-u.ac.jp

Key Words: Hepatocellular carcinoma, liver tumor, confocal laser endomicroscopy, fluorescein. standardized techniques have been established to confirm a negative liver resection margin during surgery. Therefore, surgeons are often concerned about ensuring that complete R0 tumor resection has been performed, and intraoperative histological diagnosis with frozen sections of resected specimens may be needed; however, this is a timeconsuming method, and the diagnostic accuracy of frozen sections is not satisfactory. Therefore, the development of intraoperative and real-time diagnostic techniques that assist in ensuring tumor-free margins is eagerly awaited, especially in hepatobiliary surgery.

Confocal laser endomicroscopy (CLE) provides highresolution and real-time imaging at the cellular level based upon its physical properties. The diagnostic capability of CLE has been reported in surveillance of Barrett's esophagus $(4,5)$, biliary disease (6), and colorectal disease $(7,8)$. For greater image contrast and enhancement, $10 \%$ sodium fluorescein is intravenously injected before observation under CLE (9). Although the use of fluorescein injection is generally safe (10) and beneficial, the appearance of yellowing conjunctiva and urine is unfavorable and may be misdiagnosed as icterus, especially in patients undergoing liver surgery. Recently, probe-based CLE (pCLE) was preliminarily introduced to evaluate morphological phenotypes using surgical specimens. Maki et al. (11) reported that pCLE enabled differentiation between cancerous and noncancerous tissues based on the difference in the liver autofluorescence intensity without external fluorescence administration.

Nonaka et al. (12) performed pCLE by dripping fluorescein directly onto duodenal epithelial tumors instead of injecting it intravenously and obtained favorable images for precise diagnosis. However, no reports have described the use of fluorescein-dripping method in conjunction with pCLE for liver specimens. Therefore, an ex vivo pilot study was performed using pCLE with the fluorescein-dripping method for resected liver specimens. The aim of the current study was to optimize pCLE with the fluorescein-dripping method for liver tumors and evaluate the capability of 
discrimination between cancerous and noncancerous regions using this technique.

\section{Patients and Methods}

Patients. A prospective case series protocol was performed from February to May 2017. Ten patients who underwent liver resection for liver tumors were enrolled in the pilot study. The study was approved by the institutional review board of Tottori University Hospital (1702A189).

pCLE with fluorescein-dripping method. pCLE imaging was performed using a pCLE system (Cellvizio 100 Series; Mauna Kea Technologies, Paris, France) consisting of a pCLE miniprobe connected to a laser scanning unit. The miniprobe used in this study is reusable and made for demonstration use, and with the exception of the probe length, it has the same technical specifications as the AlveoFlex probe (Mauna Kea Technologies). The laser scanning unit is a software-operated opto-electronic device with a laser source emitting at $488 \mathrm{~nm}$. The microscope uses focused laser light of the defined wavelength and passes it through a confocal aperture. The laser beam is transported via confocal miniprobes, and a distal lens sequentially scans it through a bundle of more than 10,000 optical fibers. Images are reconstructed in two dimensions. The maximum field of view is $600 \mu \mathrm{m}$. The depth of confocal observation is 0 to $50 \mu \mathrm{m}$, and the lateral resolution is $3.5 \mu \mathrm{m}$. After starting the operation, three drops of fluorescein sodium (Alcon Laboratories Inc., Fort Worth, TX, USA) are diluted with $20 \mathrm{ml}$ of normal saline and placed in a syringe in preparation for dripping.

Ex vivo observation with $p C L E$. All liver surgeries were performed at the Department of Surgery, Tottori University Hospital. After liver resection, the tumor was carefully palpated and an axial section along the maximum diameter of the liver tumor was immediately obtained in the operating room. The pCLE probe was gently placed on the subjective section in a vertical orientation, and pCLE images were collected under sequential observation with or without the fluorescein-dripping method (Figure 1). The tumor and surrounding tissue were examined on the common section. After dripping the fluorescein onto the tissue, the fluorescein was adequately washed away with 500 to $1,000 \mathrm{ml}$ of normal saline.

Upon completion of imaging, the specimens underwent histopathological diagnosis by pathologists. The pCLE findings and pathological diagnosis were compared by investigators.

Evaluation. To evaluate the optimal use of fluorescein in the dripping method, observations were performed at three exposure times after fluorescein dripping $(10,30$, and $60 \mathrm{sec})$ with the initial six patients [four with HCC, one with metastatic adenocarcinoma of the colon (MAC), and one with metastatic adenocarcinoma of extrahepatic cholangiocarcinoma (MAEC)]. One sliced section of each liver specimen was split into three pieces including both the cancerous and noncancerous regions, and the fragmented section was evaluated at each of the three fluorescein exposure times. Each obtained image was then evaluated and the optimal exposure time was determined. The pCLE images were compared with the pathological diagnosis by hematoxylin and eosin staining, the distinctive findings were investigated, and the capability and accuracy of pCLE for discrimination between cancerous and noncancerous regions were evaluated.

\section{Results}

Ten patients who underwent liver resection and provided written informed consent were enrolled in this study. pCLE images were obtained from all 10 patients [7 with HCC, 1 with MAC, 1 with MAEC, and 1 with intraductal papillary neoplasm of the bile duct (IPNB)]. Evaluable images were successfully collected in all patients examined with pCLE. Table I shows the patients' characteristics. The patients comprised one woman and nine men aged 60 to 87 years (median, 76.5 years). The median tumor diameter was $3.6 \mathrm{~cm}$ (range $=1-10 \mathrm{~cm})$. The median time from resection to bedside evaluation was $22.5 \mathrm{~min}$ (range $=10-32 \mathrm{~min}$ ). Based on the pathologic examination of the fragmented slices used for pCLE, final diagnoses and negative margins were confirmed postoperatively in all cases.

The three above-mentioned fluorescein exposure times were applied in the first six patients. The outline of cells in the cancerous region gradually became more clearly enhanced as the fluorescein exposure time increased. The cancerous regions exhibited the greatest contrast and enhancement in the 60 -sec images. Figure 2 shows representative images. Therefore, $60 \mathrm{sec}$ was considered the optimal exposure time for all patients.

In cancerous regions without fluorescein dripping, nine patients (90\%) showed non-distinctive images and one patient $(10 \%)$ showed a regular arrangement of cells. This result suggests that pCLE cannot detect specific findings in the cancerous region under morphological observation without fluorescence. With fluorescein, the cancerous regions showed the specific finding of an irregular arrangement of concentrated cells in six of seven $(85.7 \%)$ patients with HCC (Figure 3). None of the noncancerous regions in the seven patients with HCC showed these findings with fluorescein. The appearance of an irregular arrangement of concentrated cells showed $85.7 \%$ sensitivity for the cancerous region in the seven patients with HCC. Additional trends among the cancerous regions in patients with HCC included overlapping of cells with one another, well-stained cytoplasm, unstained nuclei, and a high nucleus/cytoplasm ratio. The remaining one patient with $\mathrm{HCC}$, one patient with MAC, and one patient with MAEC showed an unformed granular appearance in the cancerous region with fluorescein dripping. Conversely, the one patient with IPNB showed a non-distinctive appearance in the cancerous region with or without fluorescein dripping (Figure 4).

In all patients, the noncancerous regions showed a regular arrangement of hepatocytes with the fluorescein-dripping method. Representative cases are shown in Figure 5. Disappearance of the regular arrangement of hepatocytes in the tumor and surrounding tissue indicates the border between the cancerous and noncancerous tissue. Using the fluorescein-dripping method, the borders between the 

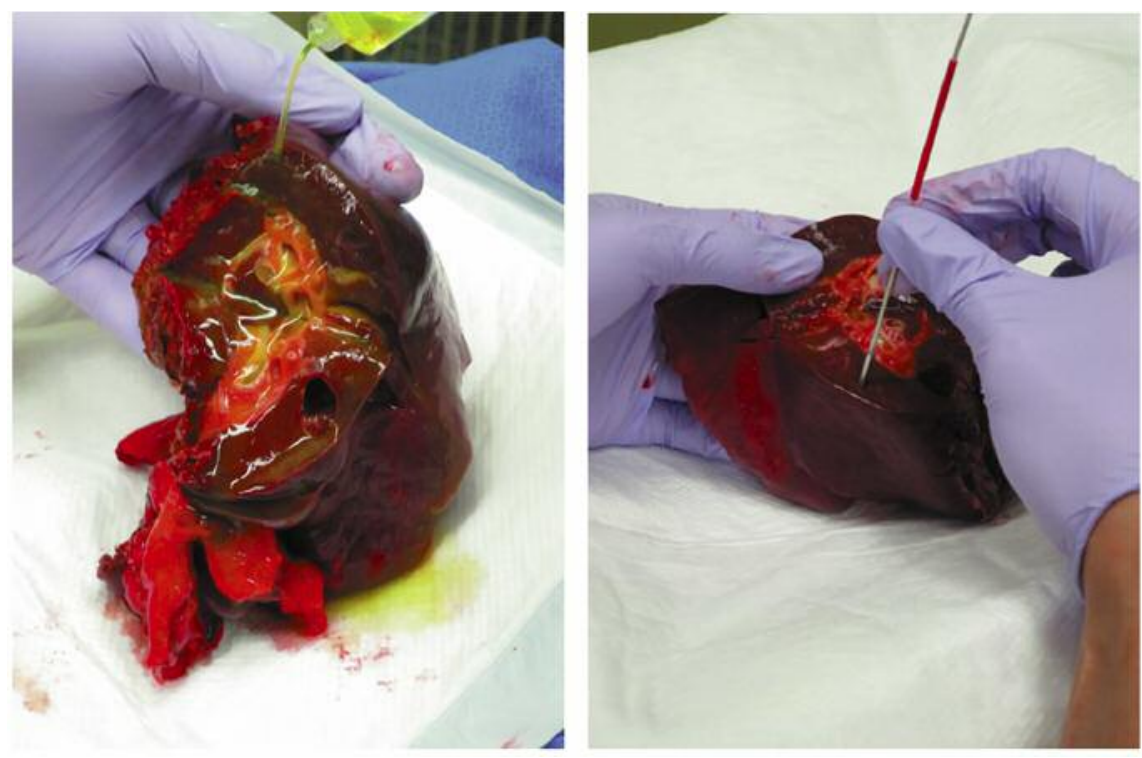

Figure 1. Fluorescein dripping and pCLE evaluation after washing with normal saline.

Table I. Characteristics of patients enrolled in this study.

\begin{tabular}{lcccccc}
\hline $\begin{array}{l}\text { Case } \\
\text { No. }\end{array}$ & $\begin{array}{c}\text { Age/ } \\
\text { Gender }\end{array}$ & $\begin{array}{c}\text { Tumor size } \\
(\mathrm{cm})\end{array}$ & $\begin{array}{c}\text { Final } \\
\text { diagnosis }\end{array}$ & Background & $\begin{array}{c}\text { Time from resection } \\
\text { to evaluation (min) }\end{array}$ & $\begin{array}{c}\text { Finding in cancerous region } \\
\text { with fluorescein dripping }\end{array}$ \\
\hline 1 & $75 / \mathrm{M}$ & 3,8 & HCC, moderately differentiated & F1-2 & 32 & IACC \\
2 & $70 / \mathrm{M}$ & 3,8 & HCC, poorly differantiated & $\mathrm{F} 3$ & 25 & IACC \\
3 & $81 / \mathrm{M}$ & 3,4 & HCC, well to moderately differntiated & $\mathrm{F} 1$ & 15 & IACC \\
4 & $78 / \mathrm{M}$ & 11 & HCC, well to moderately differentiated & $\mathrm{F} 0$ & 15 & IACC \\
5 & $75 / \mathrm{M}$ & 1,8 & HCC, well to moderately differantiated & $\mathrm{F} 1$ & 26 & Unformed granular appearance \\
6 & $84 / \mathrm{M}$ & 10 & HCC, moderately differentiated & $\mathrm{F} 1$ & 10 & IACC \\
7 & $87 / \mathrm{M}$ & 1 & HCC, well to moderately differentiated & $\mathrm{F} 3$ & 30 & IACC \\
8 & $62 / \mathrm{M}$ & 5 & MAC & F0 & 20 & Unformed granular appearance \\
9 & $60 / \mathrm{F}$ & 1,2 & MAEC & $\mathrm{F} 1$ & 18 & Unformed granular appearance \\
10 & $80 / \mathrm{M}$ & 2,5 & HCC, intestinal type & F0 & 30 & Nondistinctive \\
\hline
\end{tabular}

HCC: Hepatocellular carcinoma; MAC: metastatic adenocarcinoma of the colon; MAEC: metastatic adenocarcinoma of extrahepatic cholangiocarcinoma; IACC: irregular arrangement of concentrated cells.

cancerous and noncancerous regions could be detected by recognizing the alteration of morphological findings in all patients with HCC. Without fluorescein, the regular arrangement could be also observed by autofluorescence of the hepatocytes in the noncancerous regions of all cases. Nevertheless, the boundary between the cancerous and noncancerous regions was ambiguous without fluorescein dripping because of the absence of enhancement of the cancerous region. In addition, we had the opportunity to test another demo-probe similar to the GastroFlex probe (Mauna Kea Technologies) in some patients. The maximum field of view of this probe is $240 \mu \mathrm{m}$, and it provides higher magnification than that of the AlveoFlex probe. This probe exhibited the tumor cells in detail, but with rather low resolution (Figure 6).

\section{Discussion}

pCLE is a new diagnostic technology that can contribute to realtime observation of various tissues at the cellular level (13). It has the potential to evaluate tissue architecture at the focal plane with high-quality imaging similar to that of pathological examination during procedures such as endoscopy and surgery. A preliminary study showed that pCLE was useful for the 


\section{Non-cancerous parenchyma}
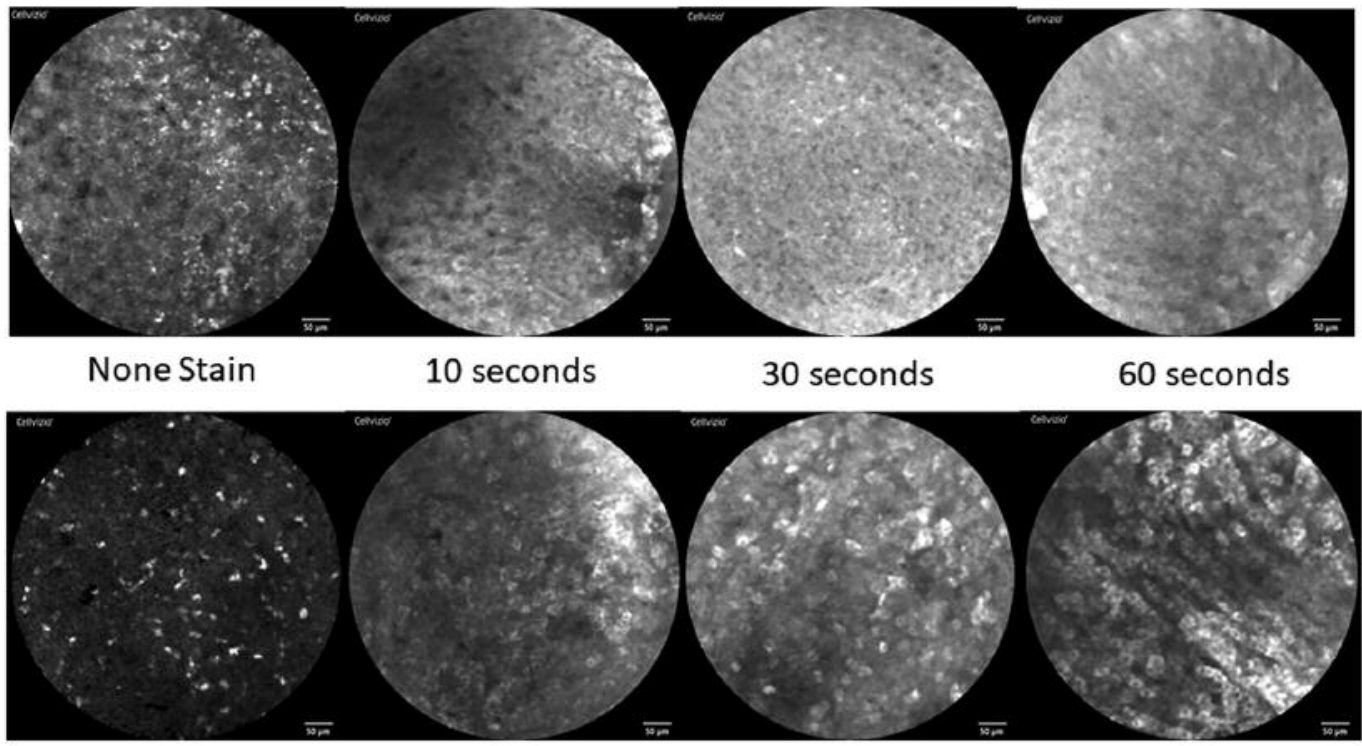

\section{Cancerous region}

Figure 2. Observation of representative images at three exposure times. Scale bar=50 $\mu \mathrm{m}$.
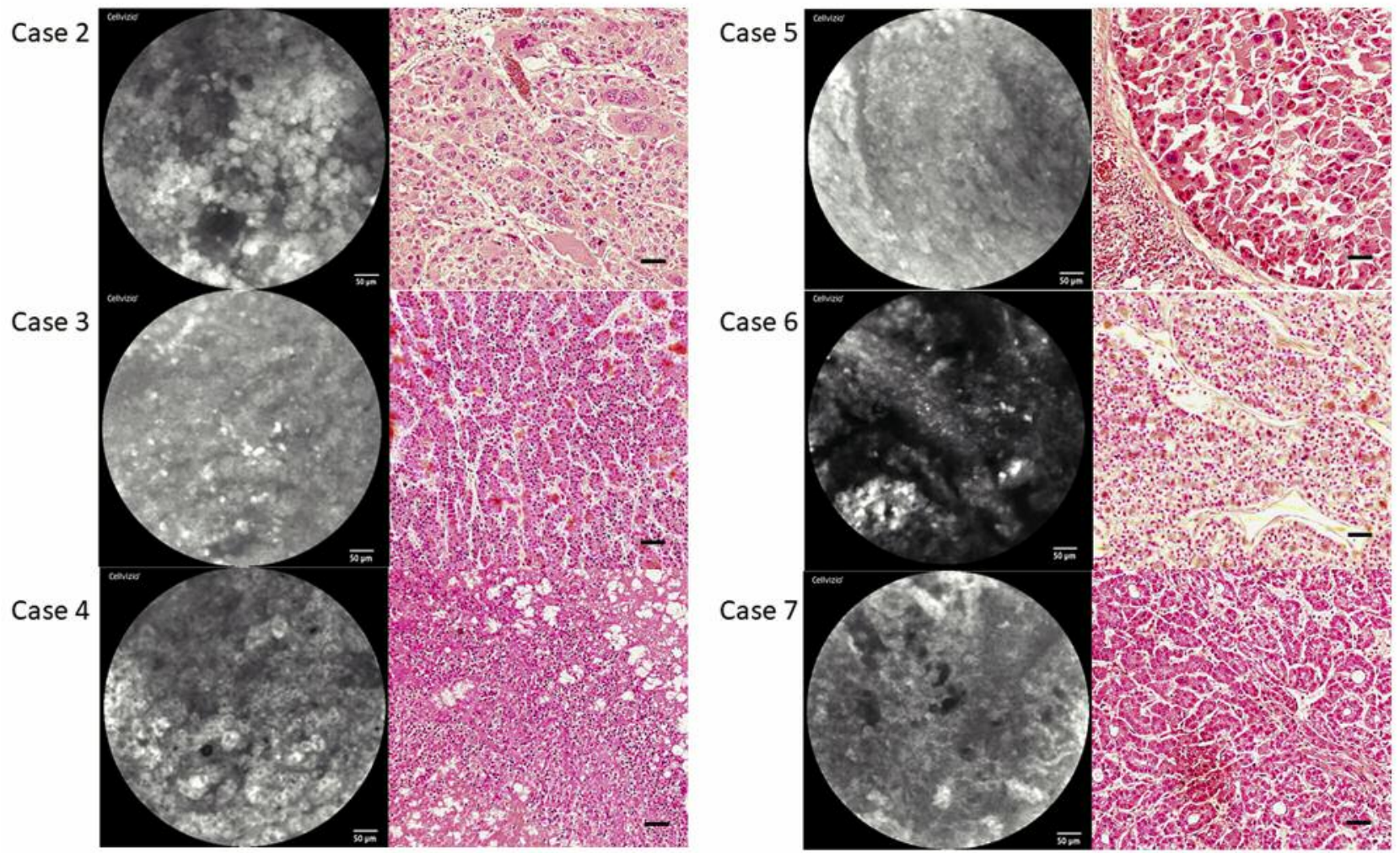

Figure 3. pCLE images with fluorescein dripping in HCC cancerous regions and the corresponding pathological examination findings. Scale bar=50 $\mu m$. 

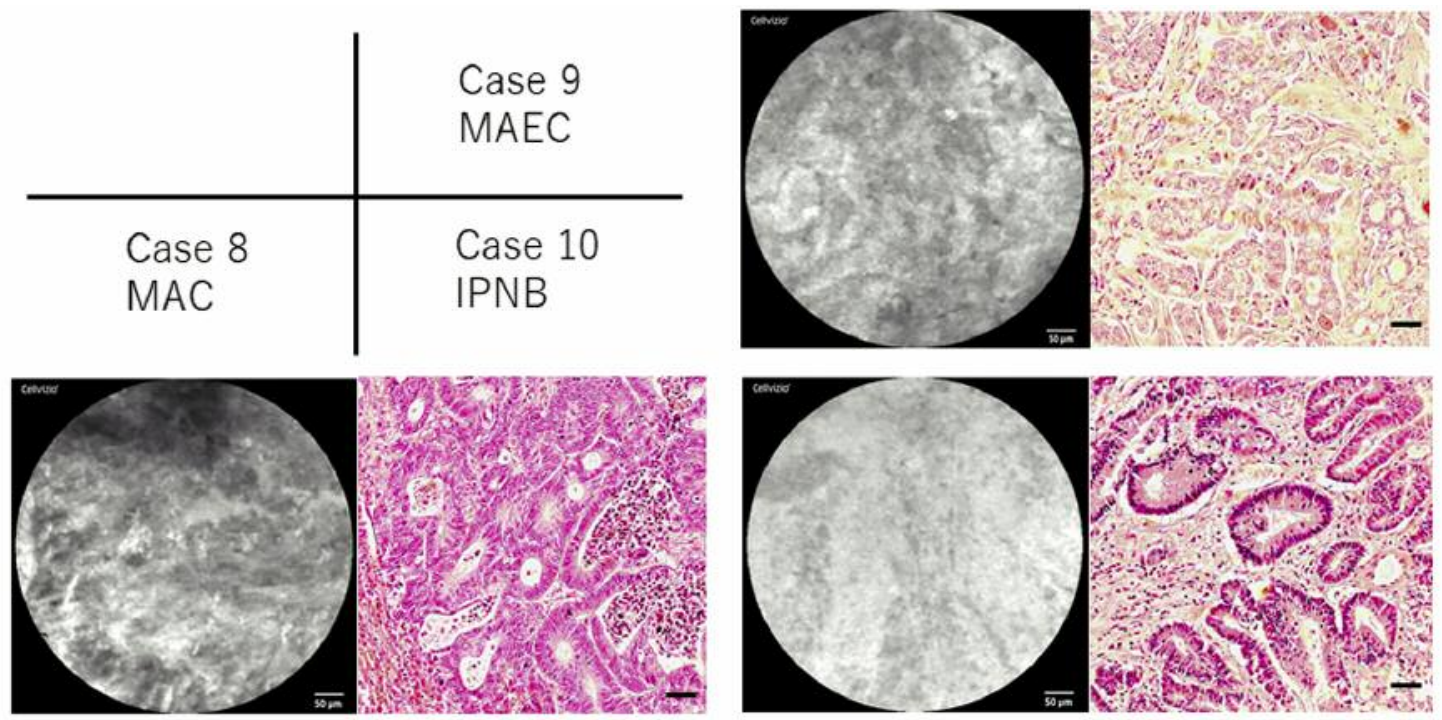

Figure 4. Other tumors except for HCC. Scale bar=50 $\mu \mathrm{m}$.
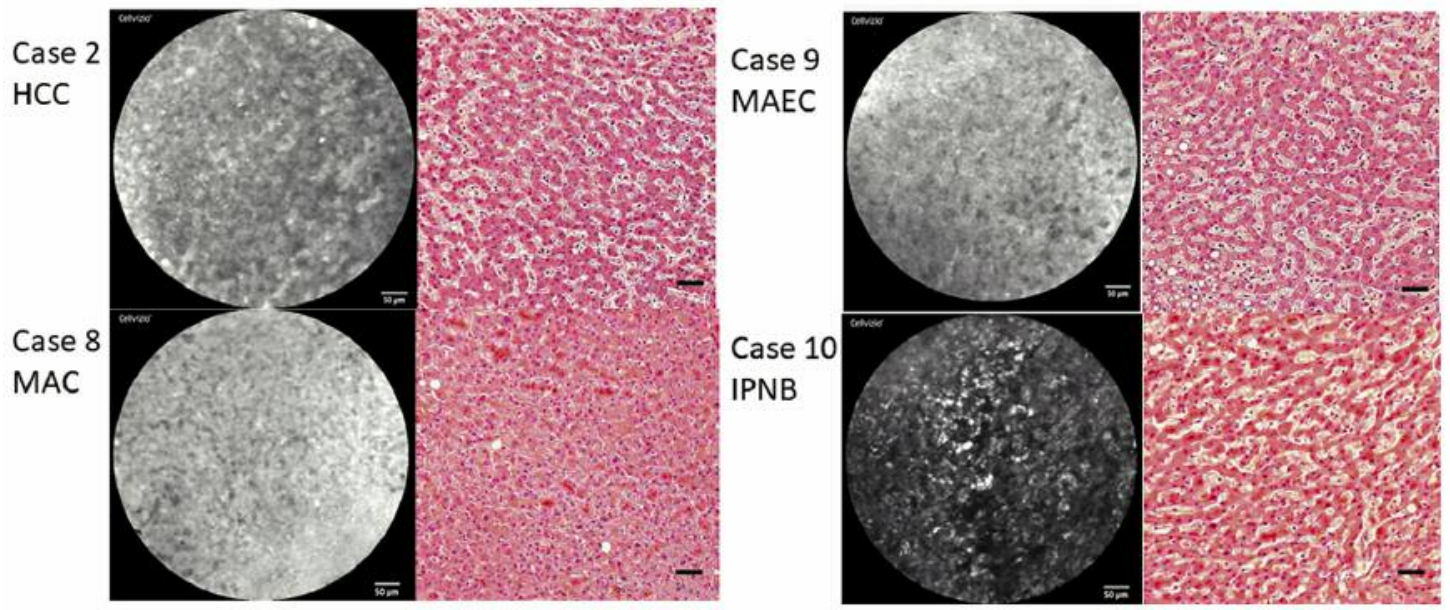

Figure 5. The noncancerous region showed a regular arrangement of hepatocytes with fluorescein dripping. Four representative cases. Scale bar=50 $\mu m$.

diagnosis of peritoneal metastasis in an animal model of laparoscopy (14). The Miami classification was developed for pCLE to distinguish normal tissue from several pathological gastrointestinal conditions and has been applied to the evaluation of Barrett's esophagus, biliary disease, colorectal disease, gastric disease, and duodenal disease (9). However, pCLE has been infrequently applied during surgery. Only a few ex vivo studies involving intravenous injection of indocyanine green (15) or without fluorescence (11) have been conducted to evaluate liver tumors. Therefore, pCLE imaging for liver tumor remains to be adequately investigated and is a developing field with no established diagnostic criteria or consensus.
Confocal imaging is based on tissue reflectance or fluorescence $(16,17)$. Fluorescent dye, which is usually administered intravenously, is required to obtain clear and distinct images (9). Fluorescein has been administered intravenously as an ophthalmological fluorescent dye since the 1960s (18). It is one of the most commonly used fluorescent materials for pCLE. Although fluorescein is generally considered non-toxic $(10,19)$, intravenous injection of fluorescein is not completely free from disadvantages. A yellowish tinge to the conjunctiva and urine is a well-known adverse effect of fluorescein injection, although the discoloration usually fades within a few days and is not 


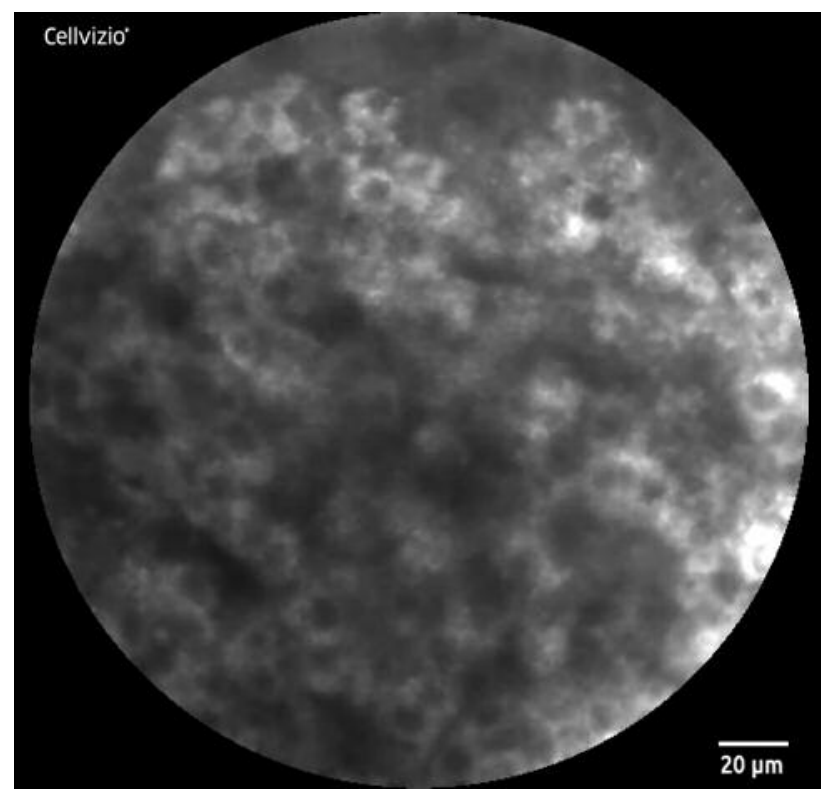

Figure 6. Another demo-probe similar to the GastroFlex probe shows HCC tumor cells with high magnification. Scale bar $=20 \mu \mathrm{m}$.

emphasized. However, it may be unfavorable especially after liver resection because it may be misdiagnosed as icterus due to liver failure. Moreover, intravenous injection of fluorescein is limited to only ophthalmological use in Japan. A few recent studies have shown that the dripping of fluorescein onto the mucosal surface during endoscopy is useful for in vivo optical diagnosis of duodenal tumors with confocal imaging $(12,20$, 21). Therefore, we devised and carried out the fluoresceindripping method for resected liver specimens in this ex vivo study. To our knowledge, no previous ex vivo studies of liver tumors using pCLE with fluorescein dripping have been performed. In the current study, the capability of discrimination between cancerous and noncancerous regions in HCC specimens was demonstrated with pCLE by the fluorescein-dripping method, which is a simple and rapid technique. First, to explore the appropriate use of fluorescein, the results at three different fluorescein exposure times were examined. The use of fluorescein in the dripping method was optimized based on these findings; specifically, the 60-sec exposure with fluorescein followed by washing with normal saline was determined to be the optimal technique. Next, 10 patients with liver tumors were observed with pCLE, and the pCLE images were compared with the pathological examination findings. The specific finding of an irregular arrangement of concentrated cells in HCC was found with good sensitivity (87\%). Furthermore, the border between cancerous and noncancerous regions could be detected in all cases of HCC with fluorescein dripping. This will contribute to real-time determination of the surgical margin in the operating room. Conversely, an irregular arrangement of concentrated cells was absent in the cancerous regions of MAC, MAEC, and IPNB. The unique imaging finding of an unformed granular appearance was present in the cancerous regions of the remaining patient with $\mathrm{HCC}$, the patient with MAC, and the patient with MAEC and may be associated with rapid proliferation of tumor cells. The imaging finding of a regular arrangement of hepatocytes, which was shown in the noncancerous regions of all 10 cases, disappeared in the cancerous region with fluorescein dripping. Therefore, this phenomenon may have great potential to distinguish between the cancerous region and the surrounding normal tissue. As mentioned above, this new ex vivo diagnostic technique for liver tumors using pCLE by the fluorescein-dripping method has the potential for both diagnosis and confirmation of negative margins.

This study has two main limitations. First, only 10 patients were examined in this pilot study, making it difficult to identify potential clinically relevant benefits. A further study with a larger number of cases is needed. Second, liver tumors are often accompanied by necrotic tissues, that may preclude the observation of characteristic findings in target regions.

In summary, pCLE is a promising procedure for the realtime diagnosis of liver pathologies when used with the fluorescein-dripping method, which was herein established for resected liver specimens. It may play a significant role in the discrimination between cancerous and noncancerous regions and in determination of safe surgical margins during surgery.

\section{Conflicts of Interest}

M. Morimoto, S. Honjo, T. Sakamoto, E. Uchinaka, M. Amisaki, Y. Arai, M. Yamamoto, Y. Fukumoto, T. Osaki, N. Tokuyasu, K. Ashida, H. Saito, and Y. Fujiwara have no conflicts of interest or financial ties to disclose.

\section{Acknowledgements}

This study received no specific grants from any funding agencies of the public, commercial, or not-for-profit sectors.

\section{References}

1 Kassebaum NJ, Bertozzi-Villa A, Coggeshall MS, Shackelford KA, Steiner C, Heuton KR, Gonzalez-Medina D, Barber R, Huynh C, Dicker D et al.: Global, regional, and national levels and causes of maternal mortality during 1990-2013: a systematic analysis for the Global Burden of Disease Study 2013. Lancet 384: 980-1004, 2014.

2 House MG, Ito H, Gonen M, Fong Y, Allen PJ, DeMatteo RP, Brennan MF, Blumgart LH, Jarnagin WR and D'Angelica MI: Survival after hepatic resection for metastatic colorectal cancer: trends in outcomes for 1,600 patients during two decades at a single institution. J Am Coll Surg 210: 744-752, 752-745, 2010. 
3 Agrawal S and Belghiti J: Oncologic resection for malignant tumors of the liver. Ann Surg 253: 656-665, 2011.

4 Sharma P, Meining AR, Coron E, Lightdale CJ, Wolfsen HC, Bansal A, Bajbouj M, Galmiche JP, Abrams JA, Rastogi A, Gupta N, Michalek JE, Lauwers GY and Wallace MB: Real-time increased detection of neoplastic tissue in Barrett's esophagus with probe-based confocal laser endomicroscopy: final results of an international multicenter, prospective, randomized, controlled trial. Gastrointest Endosc 74: 465-472, 2011.

5 Goetz M and Kiesslich R: Confocal endomicroscopy: in vivo diagnosis of neoplastic lesions of the gastrointestinal tract. Anticancer Res 28: 353-360, 2008.

6 Meining A, Saur D, Bajbouj M, Becker V, Peltier E, Hofler H, von Weyhern $\mathrm{CH}$, Schmid $\mathrm{RM}$ and Prinz $\mathrm{C}$ : In vivo histopathology for detection of gastrointestinal neoplasia with a portable, confocal miniprobe: an examiner blinded analysis. Clin Gastroenterol Hepatol 5: 1261-1267, 2007.

7 Sanduleanu S, Driessen A, Gomez-Garcia E, Hameeteman W, de Bruine A and Masclee A: In vivo diagnosis and classification of colorectal neoplasia by chromoendoscopy-guided confocal laser endomicroscopy. Clin Gastroenterol Hepatol 8: 371-378, 2010.

8 Li CQ, Xie XJ, Yu T, Gu XM, Zuo XL, Zhou CJ, Huang WQ, Chen $\mathrm{H}$ and Li YQ: Classification of inflammation activity in ulcerative colitis by confocal laser endomicroscopy. Am J Gastroenterol 105: 1391-1396, 2010.

9 Wallace M, Lauwers GY, Chen Y, Dekker E, Fockens P, Sharma $\mathrm{P}$ and Meining A: Miami classification for probe-based confocal laser endomicroscopy. Endoscopy 43: 882-891, 2011.

10 Wallace MB, Meining A, Canto MI, Fockens P, Miehlke S, Roesch T, Lightdale CJ, Pohl H, Carr-Locke D, Lohr M, Coron E, Filoche B, Giovannini M, Moreau J, Schmidt C and Kiesslich $\mathrm{R}$ : The safety of intravenous fluorescein for confocal laser endomicroscopy in the gastrointestinal tract. Aliment Pharmacol Ther 31: 548-552, 2010.

11 Maki H, Kawaguchi Y, Arita J, Akamatsu N, Kaneko J, Sakamoto Y, Hasegawa K, Harihara Y and Kokudo N: Real-time confocal laser endomicroscopic evaluation of primary liver cancer based on human liver autofluorescence. J Surg Oncol 115: 151-157, 2017.

12 Nonaka K, Ohata K, Ichihara S, Ban S, Hiejima Y, Minato Y, Tashima T, Matsuyama Y, Takita M, Matsuhashi N, Takasugi R and Neumann $\mathrm{H}$ : Development of a new classification for in vivo diagnosis of duodenal epithelial tumors with confocal laser endomicroscopy: A pilot study. Dig Endosc 28: 186-193, 2016.
13 Committee AT: Confocal laser endomicroscopy. Gastrointest Endosc 80: 928-938, 2014.

14 Hara H, Takahashi T, Nakatsuka R, Higashi S, Naka T, Sumiyama K, Miyazaki Y, Makino T, Kurokawa Y, Yamasaki M, Takiguchi S, Mori M, Doki Y and Nakajima K: A novel approach of optical biopsy using probe-based confocal laser endomicroscopy for peritoneal metastasis. Surg Endosc 30: 3437-3446, 2016.

15 Pierangelo A, Fuks D, Validire P, Benali A and Gayet B: Diagnostic accuracy of confocal laser endomicroscopy for the characterization of liver nodules. Eur J Gastroenterol Hepatol 29: 42-47, 2017.

16 Kiesslich R and Neurath MF: Endomicroscopy is born - do we still need the pathologist? Gastrointest Endosc 66: 150-153, 2007.

17 Yoshida S, Tanaka S, Hirata M, Mouri R, Kaneko I, Oka S, Yoshihara M and Chayama K: Optical biopsy of GI lesions by reflectance-type laser-scanning confocal microscopy. Gastrointestinal Endoscopy 66: 144-149, 2007.

18 Marcus DF, Bovino JA and Williams D: Adverse reactions during intravenous fluorescein angiography. Arch Ophthalmol 102: 825, 1984.

19 Moosbrugger KA and Sheidow TG: Evaluation of the side effects and image quality during fluorescein angiography comparing $2 \mathrm{~mL}$ and $5 \mathrm{~mL}$ sodium fluorescein. Can J Ophthalmol 43: 571-575, 2008.

20 Nonaka K, Ohata K and Nakai Y: Probe-based confocal laser endomicroscopy of the duodenal mucosa with fluorescein dispersion. Dig Endosc 26: 604, 2014.

21 Ohata K, Nonaka K, Ban S and Matsuhashi N: Gastroenterology: Simultaneous practice of narrow band imaging and confocal laser endomicroscopy for a case of early duodenal cancer. J Gastroenterol Hepatol 30: 966, 2015.
Received June 4, 2018

Revised June 13, 2018

Accepted June 15, 2018 